\title{
Descripción Anatómica de la Arteria Polar Renal Accesoria y su Relación con la Hipertensión Arterial
}

\author{
Anatomical Description of Accessory Renal Polar Artery \\ and its Relationship with Arterial Hypertension
}

Juan Silva ${ }^{1-2}$; Claudia Araya ${ }^{1}$; Alexis Latín ${ }^{1}$; Carolina Sambucetti ${ }^{3}$; Samara Liberona ${ }^{3}$ \& Sergio Araya $^{4}$

SILVA, J.; ARAYA, C.; LATÍN, A.; SAMBUCETTI, C.; LIBERONA, S. \& ARAYA, S. Descripción anatómica de la arteria polar renal accesoria y su relación con la hipertensión arterial. Int. J. Morphol., 38(2):336-339, 2020.

RESUMEN: La irrigación renal incluye la arteria renal y las arterias renales accesorias. Uno de estos vasos accesorios es la arteria polar renal superior o inferior. Estos vasos tienen un desarrollo embrionario y un origen anatómico similar a la arteria renal, tanto en la aorta abdominal como en sus ramas. Irrigan los segmentos renales correspondientes y se ha observado que su obliteración puede ser causa de aumento de la presión arterial, al estimular un aumento de renina circulante. El objetivo de este trabajo, es presentar un reporte de caso clínico de paciente masculino de 24 años, sin antecedentes mórbidos. Consulta por un cuadro de cefalea y mareos de 2 semanas de evolución. Clínicamente se pesquisó aumento de presión arterial y obesidad, sin fiebre ni otros hallazgos significativos. Los exámenes complementarios arrojan las siguientes alteraciones: triglicéridos (aumentados), presión arterial (aumentada), renina (aumentada) y AngioTac de abdomen evidenció una arteria renal polar accesoria superior izquierda, con estenosis vascular. La literatura indica que la hipertensión arterial esta correlacionada con la obstrucción parcial o total de una arteria renal o de una rama accesoria. Esta alteración en la disminución del flujo sanguíneo podría estimular la liberación de renina al torrente sanguíneo, afectando la presión arterial. Nuestros hallazgos concuerdan con lo descrito en la literatura y requieren ser considerados en el diagnóstico de hipertensión arterial. Este caso evidencia la necesidad de realizar una revisión exhaustiva de la base anatómica de la medicina con un enfoque integrativo, lo que permitirá mejorar el diagnóstico, el tratamiento y el pronóstico de patologías donde esta información es poco clara o inexistente.

PALABRAS CLAVE: Riñón; Irrigación renal; Presión arterial; Hipertensión arterial; Arteria polar renal accesoria.

\section{INTRODUCCIÓN}

La anatomía humana normal describe mayoritariamente una sola arteria renal $(\mathrm{AR})$ por riñón $(55,3$ $\%$ de la población). Estos vasos provienen de la aorta abdominal e ingresan al riñón por el hilio renal. Sin embargo es posible observar, variaciones de número en las arterias que aportan a la irrigación renal, sobre todo en los polos renales (15 - $30 \%$ de la población). Estas arterias han sido llamadas arterias polares renales accesorias (APRA). Embrionariamente se considera a estos vasos como arterias esplácnicas laterales, las cuales se han formado muy precozmente y persisten a lo largo de todo el desarrollo subsiguiente. En cuanto a su origen anatómico, estas ramas arteriales pueden provenir de la $\mathrm{AR}$, de las arterias ilíacas comunes, del tronco celíaco, de las arterias mesentéricas e incluso desde la aorta torácica. Dentro de las supernumerarias, se ha identificado una APRA superior (Fig.1a), normalmente es una rama aórtica que penetra en el riñón en su polo superior y que se ha observado en el 6,8 \% de los casos. También se ha descrito más comúnmente en hombres y al lado derecho. (Geyer \& Poutasse, 1962; Williams \& Warwick, 1985; Rouvière \& Delmas, 1999; Urban et al., 2001; Latarjet \& Ruiz Liard, 2004; Olave et al., 2007; Chávez et al., 2008; Raheem et al., 2008; Uflacker, 2009; Krishnaveni \& Kulkarni, 2012; Rodriguez et al., 2012; Abba et al., 2013; Amin et al., 2013; Méndez López et al., 2014).

La importancia fisiológica de la irrigación renal no solo radica en el proceso de filtración de la sangre y eliminación de desechos metabólicos, sino también en la regulación de los mecanismos que determinan la presión arterial sistémica (PA), a través del sistema renina - angiotensina - aldosterona. Este sistema determina por ejemplo, que cuando el flujo renal de sangre se encuentra disminuido por la obstrucción parcial o

\footnotetext{
${ }^{1}$ Facultad de Medicina y Ciencia, Universidad San Sebastián, Chile.

${ }^{2}$ Departamento de Anatomía y Medicina Legal, Facultad de Medicina, Universidad de Chile, Chile.

${ }^{3}$ Facultad de Ciencias para el cuidado de la Salud, Universidad San Sebastian, Chile.

${ }^{4}$ Médico internista, práctica privada, Chile.
} 
total del flujo sanguíneo renal, se libera renina al torrente circulatorio, aumentando la PA. Las observaciones experimentales han corroborado este hecho (Goldblatt et al., 1934; Kumar et al., 2015).

La hipertensión arterial (HA) corresponde a la elevación persistente de la PA sobre los límites normales, que la AHA (American Heart Association, 2018) ha definido en PAS > $130 \mathrm{mmHg}$ y PAD > $80 \mathrm{mmHg}$, donde PAS corresponde a la PA sistólica y PAD a la diastólica. (Gijón-Conde et al., 2018).

En la literatura se ha descrito una relación entre la presencia de APRA y la HA, pero ha recibido poca atención como factor etiológico de importancia en la HA. Pese a esto, es bastante común la presencia de múltiples arterias renales en pacientes hipertensos en comparación con pacientes normotensos, la razón de esta relación estaría asociada a que la obstrucción parcial o completa de estas arterias polares renales accesorias limitarían la perfusión efectiva en sus respectivos segmentos renales (Marshall, 1951; Kem et al., 2005). También se ha descrito una relación entre hipertensión e isquemia renal debido a estenosis de un vaso aberrante (Derrick \& Tyson, 1961; García Gómez et al., 2002; Ferreira Arquez, 2014).

Estos datos, parecen indicar que el conocimiento preciso de la presencia de estas variantes no solo es importante en anatomía disciplinar, sino también en cirugía, medicina interna y comunitaria, debido a ser reconocidas, en algunos casos, como causa de HA. El objetivo de este trabajo, es presentar un reporte de caso clínico de HA con presencia de APRA con estenosis vascular.
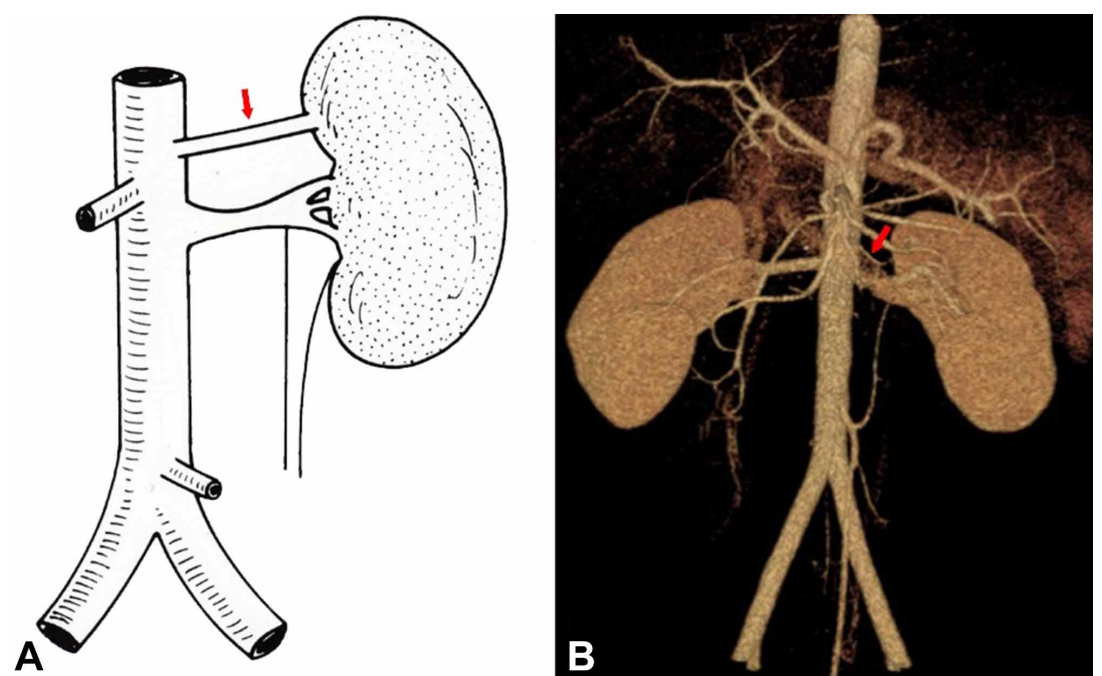

Fig. 1. En "A", (flecha roja) esquema de la disposición de una APRA superior (tomado de Uflaker, 2009). En "B", imagen reconstruida a partir de AngioTac de abdomen. En ambas imágenes e identificada con una flecha roja, hallazgo de APRA superior del lado izquierdo (visión anterior).

\section{CASO CLÍNICO}

Paciente de sexo masculino de 24 años de edad, sin antecedentes mórbidos, consulta por un cuadro de dos semanas de evolución caracterizado por cefalea y mareos. Se pesquisó aumento de PA y obesidad (IMC de 30,6; Valor normal; 18,5 - 24,9), sin fiebre ni otros hallazgos significativos. Se solicitaron exámenes complementarios de rigor, obteniéndose resultados dentro de rangos normales en el hemograma, tiempo de protrombina, orina completa, perfil hepático, perfil bioquímico, pruebas tiroideas, electrocardiograma y ecocardiografía doppler renal. En cambio, se obtuvieron resultados alterados en los siguientes exámenes:

Perfil lipídico: colesterol total $197 \mathrm{mg} / \mathrm{dL}$ (Valor normal <200 mg/dL), HDL $31 \mathrm{mg} / \mathrm{dL}$ (Valor normal; >40 mg/ dL), LDL 99 mg/dL (Valor normal; <100 mg/dL), VLDL 67 mg/dL (Valor normal; <30 mg/dL), triglicéridos 434 (Valor normal; <150 mg/dL).

Holter de Presión: promedio de presiones durante 24 horas; $139 / 78 \mathrm{mmHg}$ (Valor normal; 130/80 mmHg). Promedio de presiones diurnas; $142 / 81 \mathrm{mmHg}$ (Valor normal; 135/85 mmHg). Promedio de presiones nocturnas: 129/68 mmHg. (Valor normal 125/75 mmHg).

Pruebas hormonales: cortisol diurno; $12,2 \mu \mathrm{g} / \mathrm{dL}$ (Valor normal; 0,3 - 22,5 $\mu \mathrm{g} / \mathrm{dL}$ ), aldosterona; $126,1 \mathrm{pg} / \mathrm{mL}$ (Valor normal acostado; 30 - 160 pg/mL). Renina; 98,4 pg/ $\mathrm{mL}$ (Valor normal acostado; 4,66 - 35,6 pg/mL). Índice Aldosterona/Renina; 1,28 (Valor normal; 0,68 - 28,06).

AngioTac de abdomen: se pudo observar la presencia de una APRA izquierda que nace de la aorta abdominal por encima de la AR izquierda, con una aparente estenosis ubicada aproximadamente en la mitad de su recorrido entre su origen en la aorta abdominal y el polo superior del riñón izquierdo (Figs. 1b, 2 у 3$)$.

El análisis de los hallazgos clínicos y los resultados de los exámenes complementarios permiten concluir la presencia de hipertensión arterial en el paciente en cuestión, a causa probable de estenosis vascular de APRA superior izquierda. 


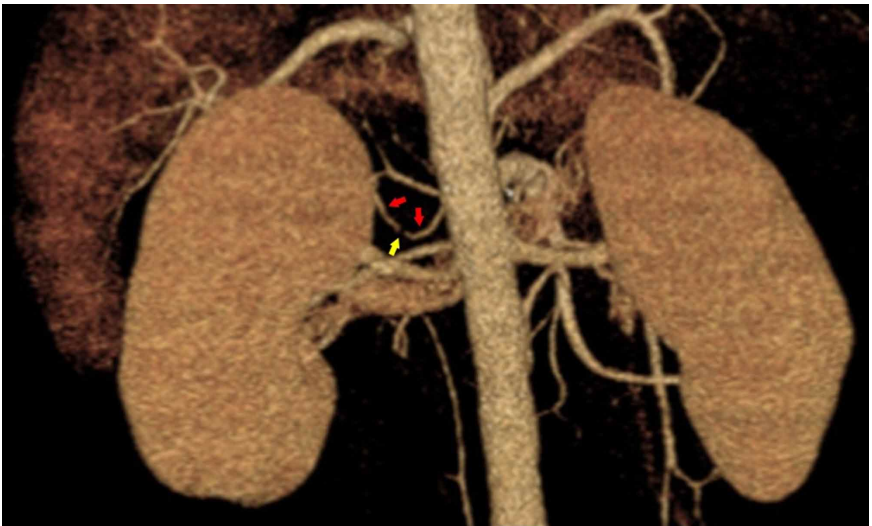

Fig. 2. En imagen reconstruida a partir de AngioTac de abdomen, se puede observar en una visión posterior, el hallazgo de una APRA superior del lado izquierdo (flechas rojas). En la flecha amarilla, se observa una estenosis en el recorrido de la citada arteria.

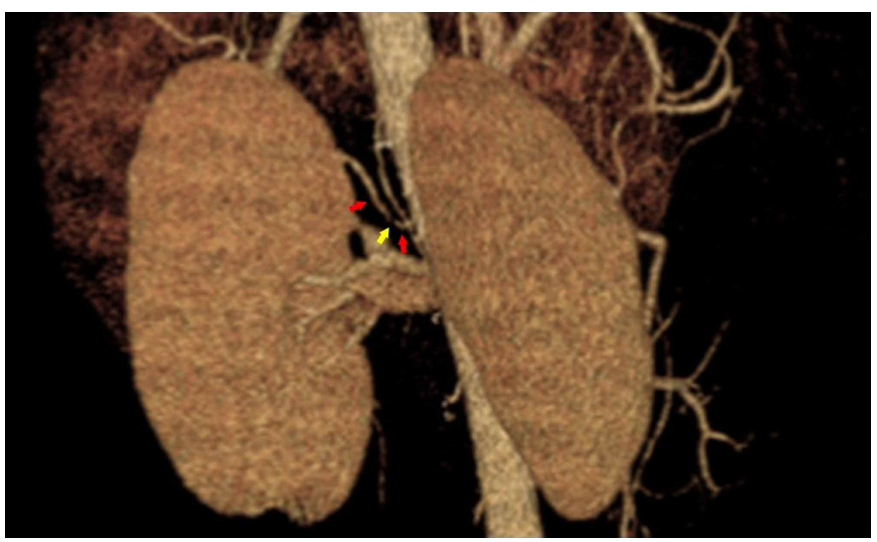

Fig. 3. En imagen reconstruida a partir de AngioTac de abdomen, se puede observar en una visión oblicua el hallazgo de una APRA superior del lado izquierdo (flechas rojas). En la flecha amarilla, se observa una estenosis en el recorrido de la citada arteria.

\section{DISCUSIÓN}

La descripción morfofuncional del riñón establece 5 segmentos renales principales. En estos segmentos no es posible observar anastomosis arteriales importantes. Tanto la AR como las APRA, presentan una relación semejante con el parénquima renal y por lo tanto con el sistema de células yuxtaglomerulares del segmento que tributan. En consecuencia, toda alteración en la disminución del flujo sanguíneo que se produzca en una APRA, ya sea superior o inferior, podría estimular la liberación de renina al torrente sanguíneo afectando en consecuencia, la PA (Derrick \& Tyson; Zahoi et al., 2015).

Se describe en la literatura, que en algunos casos, la HA estaría correlacionada con la presencia y obstrucción parcial o total de una APRA, y que además podría provocar zonas de isquemia renal segmentaria o infartos del segmento renal tribu- tado, implicando la alteración en la velocidad de filtración glomerular y como consecuencia la disminución de la función renal (Derrick \& Tyson; Koeppen \& Stanton, 2009; Kumar et al.).

Entre las patologías más frecuente que sufren las AR (enfermedades renovasculares), y que disminuyen el flujo sanguíneo, se encuentran la ateroesclerosis y la displasia fibromuscular. Pero no existen antecedentes descritos en la literatura sobre las patologías más frecuentes de las APRA (Safian \& Textor, 2001; Kumar et al; Roessler Bonzi \& Criollo Miranda, 2016), así como tampoco existen datos de prevalencia de estas arterias accesorias renales en población chilena. Considerando que el origen embrionario tanto de la AR como de sus vasos renales accesorios es semejante, podría extrapolarse que, patologías sufridas por las primeras, podrían presentarse en las segundas (Cruzat \& Olave, 2013).

Los hallazgos observados en el presente caso clínico, concuerdan con lo descrito en la literatura y requieren ser considerados en el diagnóstico clínico de HA.

\section{CONCLUSIÓN}

La literatura indica, que una de las posibles causas de la HA es la obstrucción parcial o total, ya sea de la AR como de alguna de sus ramas accesorias. En población chilena no existe estudio alguno que permita evidenciar la relación entre estas arterias accesorias y la presencia de HA.

Este caso evidencia la necesidad de realizar una nueva revisión exhaustiva de la base anatómica de la medicina y la cirugía, con un enfoque integrativo y con el claro objeto de actualizar este conocimiento fundamental, aportando información morfológica imprescindible para el diagnóstico, tratamiento y pronóstico, sobre todo en aquellas patologías donde esta información es poco clara o inexistente.

SILVA, J.; ARAYA, C.; LATÍN, A.; SAMBUCETTI, C.; LIBERONA, S. \& ARAYA, S. Anatomical description of accessory renal polar artery and its relationship with arterial hypertension. Case report. Int. J. Morphol., 38(2):336-339, 2020.

SUMMARY: The kidney irrigation includes the renal artery and the accessory renal arteries. One of these accessory vessels is the superior or inferior renal polar artery. These vessels have an embryonic development and an anatomical origin similar to the renal artery, both in the abdominal aorta but also of 
its branches. Irrigate the corresponding renal segments and it has been observed that their obliteration may cause increased blood pressure, by stimulating an increase in circulating renin. The objective of this work is to present a clinical case report of a 24year-old male patient, with no morbid history. Consultation due to headache and dizziness symptoms with 2 weeks of evolution. Clinically increased blood pressure and obesity, without fever or other significant findings. Corresponding exams showed the following changes: triglycerides (increased), blood pressure (increased), renin (increased) and abdominal AngioTac showed a left upper accessory polar renal artery with vascular stenosis. The literature indicates that arterial hypertension is correlated with partial or total obstruction of a renal artery or an accessory branch. This alteration in the decrease in blood flow could stimulate the release of renin into the bloodstream, affecting blood pressure. Our findings agree with what is described in the literature and need to be considered in the diagnosis of arterial hypertension. This case demonstrates the need for an exhaustive review of the anatomical basis of medicine with an integrative approach. This would improve diagnosis, treatment and prognosis of pathologies where this information is unclear or non-existent.

KEY WORDS: Kidney; Renal irrigation; Blood pressure; Arterial hypertension; Accessory renal polar artery.

\section{REFERENCIAS BIBLIOGRÁFICAS}

Abba, S.; Tesfay A. \& Ekanem, P. Accesory renal artery and its clinical significance. Int. J. Anat. Res., 1(3):155-7, 2013.

Amin, A.; Sharif, N. \& Tahir, M. Aberrant renal arteries and their clinical significance. Biomedica, 29:108-11, 2013.

Chávez, M.; Centeno, G.; Cesín, S. \& Antonetti, C. Consideraciones anatómicas de las arterias polares renales. Rev. Soc. Venez. Cienc. Morfol., 14(1), 2008. Disponible en: http://saber.ucv.ve/ojs/index.php/rev_svcm/ article/view/2349/2239

Cruzat, C. \& Olave, E. Renal irrigation: multiplicity of arteries. Int. J. Morphol., 31(3):911-4, 2013.

Derrick, J. R. \& Tyson, K. R. T. The surgical significance of aberrant renal arteries in relation to systemic hypertension. Circulation, 24:1192-6, 1961.

Ferreira Arquez, H. Variaciones anatómicas bilaterales de vasos renales y testiculares. Rev. CES Med., 28(2):273-81, 2014.

García Gómez, M. C.; Fernández Fresnedo, G.; Sanz De Castro, S. \& Arias, M. Hipertensión arterial renovascular secundaria a estenosis de arteria polar. Nefrología, 22(1):85-6, 2002.

Geyer, J. R. \& Poutasse, E. F. Incidence of multiple renal arteries on aortography. report of a series of 400 patients, 381 of whom had arterial hypertension. JAMA, 182:120-5, 1962.

Gijón-Conde, T.; Gorostidi, M.; Camafort, M.; Abad-Cardiel, M.; MartinRioboo, E.; Morales-Olivas, F.; Vinyoles, E.; Armario, P.; Banegas, J. R.; Coca, A.; et al. Documento de la Sociedad Española de HipertensiónLiga Española para la Lucha contra la Hipertensión Arterial (SEHLELHA) sobre las guías ACC/AHA 2017 de hipertensión arterial. Hipertens. Riesgo Vasc. 35(3):119-29, 2018.

Goldblatt, H.; Lynch, J.; Hanzal, R. F. \& Summerville, W. W. Studies on experimental hypertension : I. The production of persistent elevation of systolic blood pressure by means of renal ischemia. J. Exp. Med., 59(3):347-79, 1934.

Kem, D. C.; Lyons, D. F.; Wenzl, J.; Halverstadt, D. \& Yu, X. Renindependent hypertension caused by nonfocal stenotic aberrant renal arteries: proof of a new syndrome. Hypertension, 46(2):380-5, 2005.

Koeppen, B. \& Stanton, B. Berne y Levi Fisiología. $6^{\mathrm{a}}$ ed. Barcelona, Elsevier, 2009.

Krishnaveni, C. \& Kulkarni, R. A right ectopic kidney with bilateral multiple anomalies of the renal vasculature - A case report. J. Clin. Diagn. Res., 7(1):150-3, 2013.

Kumar, V.; Abbas, A. \& Aster, J. Robbins y Cotran, Patología Estructural y Funcional. $9^{\text {a }}$ ed. Barcelona, Elsevier, 2015.

Latarjet, M. \& Ruiz Liard, A. Anatomía Humana. $4^{\mathrm{a}}$ ed. Buenos Aires, Médica Panamericana, 2004.

Marshall, A. G. Aberrant renal arteries and hypertension. Lancet, 2(6686):701-5, 1951

Méndez López, V. C.; Casado Méndez, P. R.; López Labrada, R.; Ferrer Magadán, C. E.; Trevín Fernández, G. \& Méndez Jiménez, O. Variantes anatómicas de las arterias polares del riñón. Rev. Med. Electron., 36(Supl. 1):720-8, 2014.

Olave, E.; Henríquez, J.; Puelma, F.; Cruzat, C. \& Soto, A. Multiple renal arteries. Int. J. Morphol., 25(4):927-30, 2007.

Raheem, O.; Glacken, P.; O’Brien, M.; Hickey, D. \& Mohan, P. A single male cadaver with multiple renal arteries. Ir. J. Med. Sci., 177(3):2657, 2008.

Rodriguez, A.; Cobeñas, R.; Cruz Gallo, J.; Salamida, A.; Larrañaga, N. \& Kozima, S. Variantes anatómicas vasculares halladas de manera incidental en estudios de tomografía computada. Rev. Argent. Radiol., 77(1):19-25, 2012.

Roessler Bonzi, E. \& Criollo Miranda, T. Enfermedad renovascular. Hipertensión, 21:5-11, 2016.

Rouvière, H. \& Delmas, A. Anatomía Humana Descriptiva, Topográfica y Funcional. $10^{\text {a }}$ ed. Barcelona, Masson, 1999.

Safian, R. D. \& Textor, S. C. Renal-artery stenosis. N. Engl. J. Med., 344(6):431-42, 2001.

Uflacker, R. Atlas de Anatomía Vascular. Un Abordaje Angiográfico. $2^{\mathrm{a}} \mathrm{ed}$. Caracas, Amolca, 2009.

Urban, B. A.; Ratner, L. E. \& Fishman, E. K. Three-dimensional volumerendered CT angiography of the renal arteries and veins: normal anatomy, variants, and clinical applications. Radiographics, 21(2):37386, 2001.

Williams, P. \& Warwick, R. Anatomía de Gray. 36ª ed. Barcelona, Salvat Editores S. A., 1985.

Za hoi, D. E.; Sztika, D. \& Da escu, E. Morphological variability of arterial sources of the renal polar parenchyma and its clinical importance. Rom. J. Morphol. Embryol., 56(4):1403-9, 2015.

Dirección para correspondencia:

Prof. Dr. Juan Luis Silva Rubilar

Facultad de Medicina y Ciencia

Universidad San Sebastián

Lota 2465. C. P. 7510157

Providencia, Santiago

CHILE

Email: juan.silva@uss.cl

Recibido: 30-07-2019

Aceptado: 23-09-2019 